\title{
REMARKS ON THE ROOT-CLUSTERING OF A POLYNOMIAL IN A CERTAIN REGION IN THE COMPLEX PLANE*
}

\author{
By E. I. JURY AND S. M. AHN (University of California, Berkeley)
}

\begin{abstract}
A general formulation for the root clustering of a polynomial is given. An attempt has been made to answer an open question raised by Kalman.
\end{abstract}

Introduction. Since 1852, when Hermite first established the connection between the number of the roots of a polynomial in an arbitrary half-plane and the signature of a certain quadratic form, the root-clustering problem has been investigated by many mathematicians, physicists and engineers. Recently Kalman [1] showed a general formulation which includes all the previously known results as particular cases. His formulation applies to the region $\Gamma$ in the complex plane given by

$$
\Gamma \triangleq\left\{\left.z \in C|| \gamma(z)\right|^{2}-|\delta(z)|^{2}>0\right\}
$$

where $\gamma(z)$ and $\delta(z)$ are two arbitrary polynomials with complex coefficients and deg $\gamma+$ $\operatorname{deg} \delta>0$.

He showed that the criteria can be expressed by rational functions of the coefficients of the given polynomial and their complex conjugates by purely algebraic way. An entirely different approach was employed for the same region $\Gamma$ by Jury and Ahn [2], where the form of positive definite matrix was shown explicitly. Furthermore, they generalized the Lyapunov equation, showing that the positive definite matrix satisfies a certain matrix equation and that the elements of this matrix are rational functions of the coefficients of the given polynomial and their complex conjugates.

Kalman [1] raised an open question as to whether $\Gamma$ is the largest class of the region in the complex plane for which the criteria can be described by only rational functions of the coefficients of the polynomial. It must be noted that $\Gamma$ includes half planes, circles, and hyperbolas but not ellipses and parabolas. In [3], Howland obtained the matrix equation for a more generalized region than $\Gamma$. However, he showed only that for a particular positive definite hermitian $Q$, there exists a positive definite hermitian $H$ (see Eq. (2)). In other words, the uniqueness of $H$ for any given $Q$ has not been shown for the region he chose, thus leaving the same question on the rationality left open.

Here we show that the criteria for the ellipses and parabolas can also be described by rational functions of the coefficients and their complex conjugates. The region described here does not encompass $\Gamma$ in [2].

Let

$$
\phi(\alpha, \beta)=C_{00}+C_{01} \beta+C_{02} \beta^{2}+C_{10} \alpha+C_{11} \alpha \beta+C_{20} \alpha^{2}
$$

where $C_{11}+\bar{C}_{11} \leq 0, \Omega=\{z \in C \mid \operatorname{Re} \phi(\bar{z}, z)>0\}, A \in C^{n \times n}, \lambda_{i}, i=1,2, \cdots, n$,

\footnotetext{
* Received November 13, 1972. Research sponsored by the National Science Foundation Grant
} GK-33347. 
are the eigenvalues of $A$, and $\Omega$ includes half-plane, circles, hyperbolas, ellipses, and parabolas.

Theorem. Let

$$
\phi\left(\bar{\lambda}_{i}, \lambda_{i}\right)+\phi\left(\lambda_{i}, \bar{\lambda}_{i}\right) \neq 0, \forall \lambda_{i}, \lambda_{i} \in \Omega, i, j=1,2, \cdots, n .
$$

Under this condition, $\lambda_{i} \in \Omega, i=1,2, \cdots n$, if and only if for all positive definite hermitian $Q \in C^{n \times n}$, there exists a unique positive definite hermitian $H \in C^{n \times n}$ such that

$$
\begin{aligned}
\left(\bar{C}_{00}+C_{00}\right) H+\left(\bar{C}_{01}+C_{10}\right) A^{*} H & +\left(\bar{C}_{02}+C_{20}\right)\left(A^{*}\right)^{2} H+\left(\bar{C}_{10}+C_{01}\right) H A \\
& +\left(\bar{C}_{11}+C_{11}\right) A^{*} H A+\left(\bar{C}_{20}+C_{02}\right) H A^{2}=Q,
\end{aligned}
$$

where ${ }^{*}$ denotes the complex conjugate transpose. Furthermore, the elements of $H$ are rational functions of the elements of $A$ and $A^{*}$.

Proof. "Only if": let $x_{K} \in C^{n}$ be the eigenvector corresponding to the eigenvalue $\lambda_{k} ;$ then

$$
\left(\phi\left(\bar{\lambda}_{K}, \lambda_{K}\right)+\bar{\phi}\left(\lambda_{K}, \bar{\lambda}_{K}\right)\right) x_{K}^{*} H x_{K}=x_{K}^{*} Q x_{K} .
$$

"If": Eq. (2) is equivalent to $n^{2}$ linear equations, whose $n^{2} \times n^{2}$ coefficient matrix has

$$
\phi\left(\bar{\lambda}_{i}, \lambda_{i}\right)+\bar{\phi}\left(\lambda_{i}, \bar{\lambda}_{i}\right), \quad i, j=1,2, \cdots, n
$$

as its eigenvalues [4]. By hypothesis, Eq. (2) has a unique solution $H$ whose elements are rational functions of the elements of $A$ and $A^{*}$. We first prove that there exists a positive definite $H_{0} \in C^{n \times n}$ and a positive definite $Q_{0} \in C^{n \times n}$ which satisfy Eq. (2).

Let $J$ be the Jordan form of $A$; then there exists a nonsingular $P \in C^{n \times n}$ such that

$$
P A P^{-1}=\Lambda+U
$$

where $\Lambda$ is diagonal and all the elements of $U$ are either 0 or 1 with all non-zero elements located on the diagonal above the main diagonal. For some small $\delta>0$, define the nonsingular matrix $D$ by

$$
D=\operatorname{diag}\left(1, \delta^{-1}, \delta^{-2}, \cdots, \delta^{-(n-1)}\right) .
$$

Then

$$
D P A P^{-1} D=\Lambda+\delta U .
$$

Now define $H_{0}=\operatorname{diag}\left(\operatorname{Re} \phi\left(\bar{\lambda}_{1}, \lambda_{1}\right), \operatorname{Re} \phi\left(\bar{\lambda}_{2}, \lambda_{2}\right) \cdots, \operatorname{Re} \phi\left(\bar{\lambda}_{n}, \lambda_{n}\right)\right)$. Then $Q_{0}$ defined by Eq. (2) is positive definite by Gershgorin's theorem [4]. For arbitrary positive definite $Q \in C^{n \times n}$ let $Q_{t}=t Q+(1-t) Q_{0}$, where $0 \leq t \leq 1$. Clearly $Q_{t}$ is positive definite. Eq. (2) has a unique solution $H_{t} \in C^{n \times n}$ for $Q=Q_{t}$. The eigenvalues of $H_{t}$ are real and vary continuously with $t$. Hence if we prove that $H_{t}$ never becomes singular, we are done. Let

$$
H_{t}=\left[\begin{array}{c:c}
\hat{H}_{t} & 0 \\
\hdashline 0 & 0
\end{array}\right] ;
$$

we substitute $H=H_{t}$ in Eq. (2) and arrive at a contradiction to the fact that $Q$ is positive definite using the hypothesis $C_{11}+\bar{C}_{11} \leq 0$.

Q.E.D.

Example 1 (Ellipse). Let $\phi(\alpha, \beta)=-2 \alpha \beta-\alpha^{2}+1 . A \in C^{n \times n}$ has all its eigen- 
values inside the ellipse $3 x^{2}+y^{2}-1<0$ if and only if for all positive definite hermitian $Q \in C^{n \times n}$ there exists a unique positive definite hermitian $H \in C^{n \times n}$ such that

$$
2 H-\left(A^{*}\right)^{2} H-4 A^{*} H A-H A^{2}=Q
$$

and the elements of $H$ are rational functions of the elements of $A$ and $A^{*}$.

Proof. We have only to show that (1) holds, that is, all $\lambda_{1} \triangleq x_{1}+i y_{1}$ and $\lambda_{2} \triangleq$ $x_{2}+i y_{2}$ belonging to the ellipse satisfy

$$
-4 \bar{\lambda}_{1} \lambda_{2}-\bar{\lambda}_{1}^{2}-{\lambda_{2}}^{2}+2 \neq 0 \text {. }
$$

Suppose the equality holds; then $x_{1}\left(2 y_{2}-y_{1}\right)=x_{2}\left(2 y_{1}-y_{2}\right)$ and

$$
4 x_{1} x_{2}+4 y_{1} y_{2}+{x_{1}}^{2}-{y_{1}}^{2}+{x_{2}}^{2}-{y_{2}}^{2}-2=0 \text {. }
$$

Let

$$
x_{1} / x_{2}=\left(2 y_{1}-y_{2}\right) /\left(-y_{1}+2 y_{2}\right) \triangleq k
$$

Simple calculation shows $k$ is well defined. Substituting (4) into (3), we obtain

$$
\frac{-5 k^{2}+4 k+1}{(2+k)^{2}} y_{2}{ }^{2}+\frac{5 k^{2}-4 k-1}{k^{2}+4 k+1}<0
$$

and

$$
\frac{k^{2}+4 k-5}{(2+k)^{2}} y_{2}^{2}+\frac{-k^{2}-4 k+5}{k^{2}+4 k+1}<0 .
$$

In the region $k \leq-5$ and $k \geq-0.5$, (5) and (6) contradict to each other. In the region $-5<k<-0.5, y_{2}^{2} \geq 1$, which is also a contradiction.

Example 2 (Parabola). Let $\phi(\alpha, \beta)=\alpha^{2}+2 \alpha-\alpha \beta$; then the parabolic region is given by $y^{2}<x$ and the matrix equation is

$$
H A^{2}-2 A^{*} H A+\left(A^{*}\right)^{2} H+2 H A+2\left(A^{*}\right)^{2} H=Q .
$$

A proof similar to that given to Example 1 follows.

Conclusion. Needless to say, root-clustering problems are amongst the oldest ones in mathematics and still are extensively investigated. We have answered the open question raised by Kalman in a negative way. Hence the question still remains open "What is the largest class of regions in the complex plane where the criteria can be expressed by the rational functions of only the coefficients and their complex conjugates of the given polynomial?" We believe that additional constraints are needed for enlarging the regions in this paper or in the previous papers $[1,2]$.

\section{References}

[1] R. E. Kalmai, Algebraic characterization of polynomials whose zeros lie in certain algebraic domains, Proc. Nat. Acad. Sci. 64, 818-823 (1969)

[2] E. I. Jury and S. M. Ahn, Symmetric and innerwise matrices for the root-clustering and root-distribution of a polynomial, J. Franklin Inst. 293, 433-450. (1972)

[3] J. L. Howland, Matrix equations and the separation of matrix eigenvalues, J. Math. Anal. Appl. 33, 683691 (1971)

[4] P. Lancaster, Theory of matrices, Academic Press, New York, 1969

[5] R. D. Hill, Inertia theory for simultaneously triangulizable complex matrices, Linear Algebra and its Applications, 131-142 (1969) 